\title{
Effects of Immobilized Recombinant Human BMP-2/Collagen on Cellular Activity of Osteoblast Like Cells
}

\author{
Hidetsugu Tsujigiwa'), Hitoshi Nagatsuka1), Mehmet Gunduz'), Jin Tan²), \\ Katsunori Kanata ${ }^{3)}$, Masao Yamada ${ }^{1)}$,and Noriyuki Nagai ${ }^{1)}$
}

\author{
1) Okayama University, Graduate School of Medicine, Dentistry and Pharmaceutical Sciences \\ 2) Tairen University \\ 3) Japan Institute for Advanced Dentistry, Osaka
}

\begin{abstract}
The use of rhBMP-2 to induce ectopic bone formation requires a carrier. Type I atelocollagen, a biomaterial with a porous structure, excellent operational features and biocompatibility, is known to be an effective carrier for rhBMP-2. However, conventionally used lyophilized rhBMP-2/collagen mixture does not necessarily give adequate bone induction effect. In the present study, we examined the effect of immobilizing rhBMP-2 to type I atelocollagen on the cellular activity of ST2 cells. The following results were obtained: (1) rhBMP-2 was effectively immobilized to succinylated type I atelocollagen, indicating the usefulness of succinylated type I atelocollagen in immobilization. (2) Studies of alkaline phosphatase activity confirmed the effectiveness of rhBMP-2 immobilized on succinylated in augmenting cellular activity.
\end{abstract}

Key words: BMP, collagen Type 1, immobilization, alkaline phosphatase, ST2cells

\section{Introduction}

BMP-2 possesses the strongest bone inducing activity, and basic research has been conducted aiming at clinical application of this protein. A carrier is indispensable when using rhBMP-2 to induce ectopic bone formation. Basic research has indicated that type I atelocollagen, a biomaterial with a porous structure and biocompatibility is highly effective as a carrier for rhBMP-2. However, these studies used a lyophilized rhBMP-2/collagen mixture, which may not possess adequate bone induction effect. The mechanism of action of immobilized rhBMP-2 on cells is based on the theories of biomaterial science with respect to chemically bonding the ligand rhBMP-2 to a carrier. The ligandcarrier composite will react with the receptors on target cells and initiate the cellular function control mechanism. When growth factors such as insulin like growth factor and epithelial cell growth factor are immobilized to a carrier, the immobilized growth factor binds with the receptor on the cell surface forming a complex. Without being internalized into the cell, the growth factor-receptor complex is able to transduce signals into the cell over a long period of time, achieving high efficiency in promoting cell proliferation. BMP family is considered to be differentiation factors. However, the effects of immobilized rhBMP-2 on cell differentiation and augmentation of cellular activity remain unknown. The purpose of this study was to test the hypothesis that chemically bonding rhBMP-2 to type I atelocollagen may increase the bone induction effect. Immobilization will also have the effect of preventing the diffusion of rhBMP within the body.

\section{Cell culture}

\section{Materials and Methods}

Mouse ST2 cell line (Cell No. RCB0224, from the cell bank of the Institute of Physical and Chemical Research, Japan.) was used in this study. The cells were cultured in $\alpha$-MEM supplemented with antimicrobial agents.

Preparation of rhBMP-2/succinylated atelocollagen samples
rhBMP-2 was provided by Yamanouchi, Inc., Japan. Succinylated type I atelocollagen (provided by Koken, Inc., Japan) was used as carrier. For immobilization, 1-ethyl-3-(3dimethylaminopropyl) carbodiimide (water soluble carbodiimide [WSC] ) was used. The principle of rhBMP-2/collagen immobilization using WSC was previously established. Succinylated atelocollagen was dissolved in diluted hydrochloric acid with a $\mathrm{pH}$ of 3.0. As a pre-treatment, WSC was added to the succinylated atelocollagen solution to obtain a final concentration of $1 \mathrm{mg} / \mathrm{ml}$ and incubated at $4{ }^{\circ} \mathrm{C}$ for $2 \mathrm{~h}$. Then rhBMP-2 was added and immobilization took place at $4{ }^{\circ} \mathrm{C}$ for $24 \mathrm{~h}$.

Effect of immobilized rhBMP-2/ succinylated atelocollagen on ST2 cells

Time course of ALP activity: ST2 cells were seeded in a $10 \mathrm{~cm}$ petridish and cultured. The samples were added to growth medium such that the final atelocollagen concentration was $150 \mu \mathrm{g} / \mathrm{ml}$ and rhBMP-2 concentration was $200 \mathrm{ng} / \mathrm{ml}$. The cells were collected at days 1, 3, 5 and 7 to measure ALP activity.

ALP activity at different concentrations of rhBMP: After ST2 cells seeded in a 96-well plate reached confluent growth, the medium was completely removed. These samples were added to growth medium such that the final type I atelocollagen concentration was $150 \mu \mathrm{\gamma} / \mathrm{ml}$ and rhBMP-2 concentrations were $0,100,200,500,750$ and 1,000 ng/ml. The cells were collected at day 5 for measurement of ALP activity.

\section{Results}

Effect of immobilized rhBMP/ succinylated atelocollagen on ST2 cells

ALP activity with time course Compared to the succinylated atelocollagen control group, both the immobilized and nonimmobilized groups showed increased ALP activity with time. The immobilized and non-immobilized groups showed no differences in ALP activity on days 1 and 3, but significantly higher ALP activity was observed in the immobilized group compared 
to the non-immobilized group on days 5 and 7 (Fig. 1).

ALP activity at different rhBMP-2 concentrations

In both immobilized and non-immobilized groups, ALP activity increased with increased concentration of rhBMP-2. At all rhBMP2 concentrations, ALP activity was higher in the immobilized group compared to the non-immobilized group (Fig. 2).

\section{Conclusion}

This study suggest that immobilization of rhBMP-2 to type I atelocollagen may increase the bone-inducing effect. New immobilization methods have to be explored in the future to increase the efficiency of bone induction.

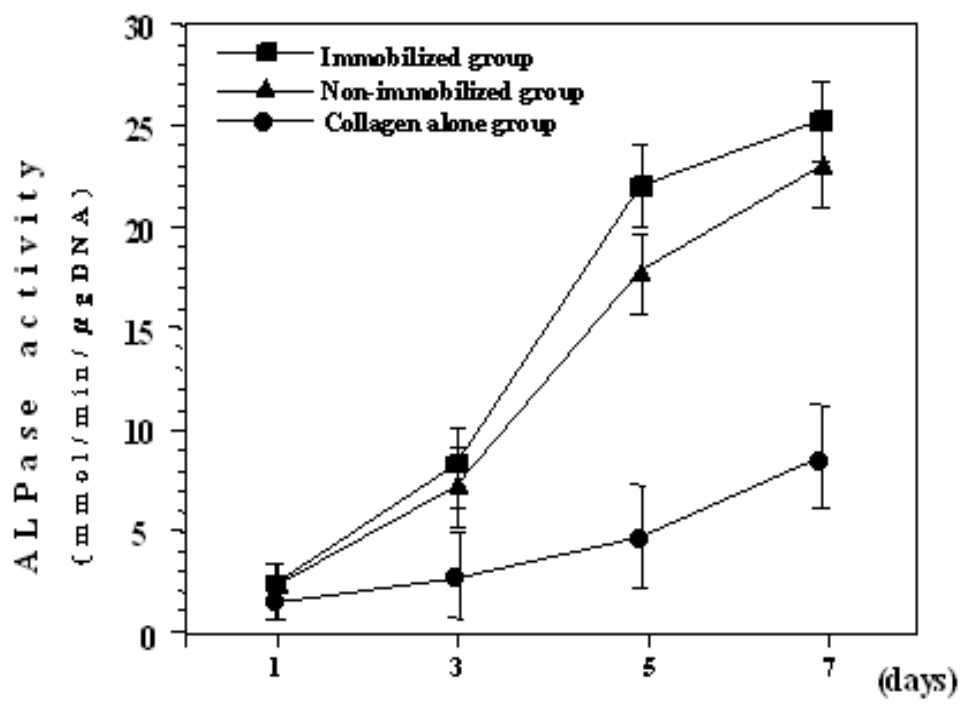

Fig.1 Changes of ALP activity in ST2 cells with time. Both the immobilized and nonimmobilized groups show increased ALP activity with time.

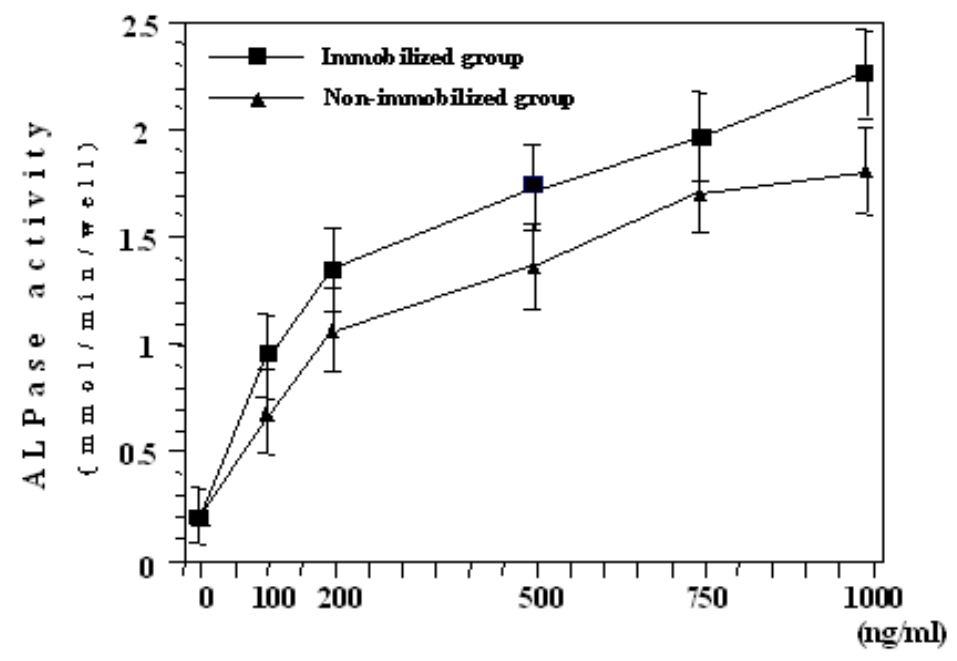

Fig.2 Changes of ALP activity in ST2 cells at various rhBMP concentrations. 